\title{
A critique on Materials for Orthodontic Aligners through sustainable Manufacturing
}

\author{
${ }^{1}$ Kalpana Kilaru \\ ${ }^{1}$ Dept. of Mechanical Engineering, KG Reddy College of Engineering \& Technology, Hyderabad, India - 500075.
}

\begin{abstract}
Materials will play an important role in any manufacturing sector. Additive manufacturing is the one of the most Emerging Manufacturing technology which is adding a sustainable dimension to Human life by using bio medical dental aligners in orthodontics. The new presentation of Sustainable manufacturing is upsetting endodontist, furthermore, it is in any event, it is applied to dental treatment of poor alignment of teeth and jaws. Exact, customized, Detachable aligners are an appropriate option in contrast to ordinary orthodontic machines, offering a more agreeable furthermore, and productive answer for patients Simultaneously, Detachable Clips are created by a thermoset's interaction utilizing different sorts of thermoset elements. The Elastomer system modifies the Characteristics of the element, and the intra oral climate moreover changes the characteristic of an unmistakable clip, influencing execution of the element. The current audit recommends the appropriateness of Additive manufactured aligners is better than that of thermoset made aligners due to earlier expanded precision, load obstruction, and lower twisting. It is a general steadier approach to produce an aligner where sub millimetre developments can have an effect in treatment result. Direct 3D printing addresses an intricate strategy to command the density of the aligner and in this manner has superior capacity to command the power angle that are utilized to create teeth development. There is presently no other endorsed material available that can make this.
\end{abstract}

Keywords : 3D printing, Clear Braces, Malocclusion Overbite, Orthodontics.

\section{Introduction}

Additive manufacturing offers the production of exceptionally exact detachable clips with delicate borders, carefully planned, indistinguishably imitated for a whole arrangement of therapy aligners, giving a superior fit, longer viability, and coherent. malocclusion overbite was treated with the utilization of metal rings established to teeth to help wires for applying moving powers. This underlying treatment methodology for the revision of dental and skeletal malocclusion overbite was regularly joined by a colossal number of dental caries since it was practically inconceivable to keep up right dental cleanliness because of the restricted contribution of dental cleanliness devices in the market around then and to the mechanical encumbrance of the established rings and resulting dental plaque arrangement on the teeth [1]. Later, the rings were subbed by the main sections to help wires produced using treated steel, which offered a few benefits, like high strength, sturdiness, decreased rubbing, improved salivary stream, less food assortment, and they were generally simple to frame. This awesome mechanical bounce addressed an upheaval and was made conceivable because of the advancement of dental science materials ready to bond the sections straightforwardly onto the teeth. The regular sections made of straightforward or clear non-metallic material were presented in the mid 1970's (Figure 1).
The principal straightforward sections made of unfilled polycarbonate were later supplanted by ceramic built up, fiberglass supported, and polycarbonate-supported sections with a metallic supplement to limit creep deformity $[1,2,3]$. Afterward, fired sections (monocrystalline sapphire and polycrystalline artistic) were utilized; notwithstanding, the hardness of those materials could cause polish scraped area [4].

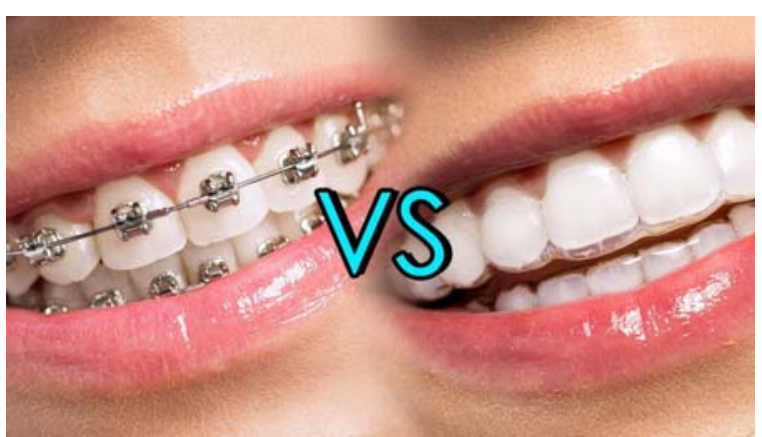

Figure 1. Traditional orthodontic treatment(a) and thermal formed clear aligner with its $3 \mathrm{D}$ printed $\operatorname{mould}(\mathbf{b})$.

Additionally, to progress in material utilized for sections, there has been a mechanical movement in the various kinds of arch wire has been utilized since the advocacy 
of orthodontic treatment. Introductory utilization of customarily treated steel arch wire was ultimately supplanted by exact optical fibre, Jacketing Teflon cable, epoxy covered cable, titanium plastic covered cable and Bio force cable $[1,4]$. This innovative improvement prompted the third unrest and addresses current contemporary orthodontic science.

\subsection{Historical development of Dental Aligners in additive manufacturing}

Towards the finish of the most recent century and in the principal ten years of this century, the initial clips for dental purposes seemed [1,5]. This progressive improvement was primarily determined by the pressing factor of patients looking for more agreeable and less meddling orthodontic strategies for their teeth. Surely, since their presentation as a treatment choice in orthodontics, aligners have delighted in extraordinary fame and the number of patients going through dental treatment with detachable clips has just expanded. The utilization of detachable clips in orthodontia has made a change in perspective for endodontist and has empowered individuals of every age, including more established grown-ups, to look for treatment for their malocclusion. [1]

The point in the current story audit is explains about the survey related to historical backdrop of clear aligners' assembling measures from thermoforming to $3 \mathrm{D}$ direct printing; to talk about the benefits of 3D printed aligners in contrast with past advances, investigate the condition of the craftsmanship, incorporate potential places of analysis like a current absence of endorsed and advertised materials and restricted presence of programming bundles and propose where we need to go from the creators' viewpoint. Ultimately, extraordinary consideration will be paid to the expected cytotoxicity of printable pitches, and it establishes the framework for future viewpoints and choices.

\section{Major improvements of orthodontics in additive manufacturing}

As recently depicted - similarly as the relocate from endodontic rings to sections was made conceivable direct propels in endodontic elements; the utilization of detachable clips will spike the innovative work of new elements explicitly for use in additive manufacturing [1]. Sustainable manufacturing, otherwise called added substance producing, traces all the way back to the 1980s and is presently arriving at degree of development where it is progressively entity used in the fields of endodontic also, clinical displaying. Come into being various applications in oral medical procedure, endodontics, therapeutic dentistry, prosthodontics, implant theory and element producing [1,6]. Additive manufacturing empowers assembling of elements film by film rather than regular assembling techniques that depend on assembling, shaping, and accumulative strategies [7]. Present elements utilized for additional manufacturing imprinting in endodontics incorporate acrylonitrilebutadienestyrene plastic, stereolithography materials (epoxy pitches), polylactic corrosive, polyamide (nylon), glass-filled polyamide, silver, steel, titanium, photopolymers, wax, and polycarbonate $[1,8]$.

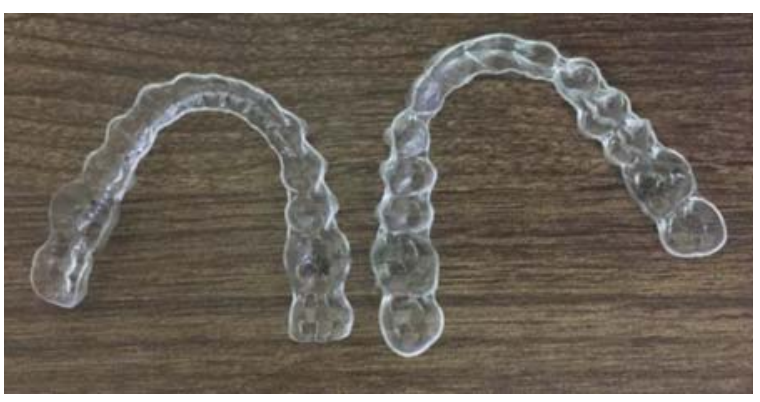

Figure 2. Three-Dimensional printed clear Orthodontic aligner.

An illustration of the use of $3 \mathrm{D}$ imprinting the creation of detachable clips is the previously mentioned additive manufacturing of endodontic models for the reasonable detachable clips shaping. In view utilization of $3 \mathrm{D}$ printed models was the initial move about simplification of blunders and errors (e.g., mathematical mistakes) happening during impression assortment $[1,9]$. Maybe than utilizing blunder inclined mortar models that are checked and displayed to create different arrangement stages, it is better than utilize advanced impression taking and 3D printing for development as shown in Fig. 2. Utilization of a reasonable detachable clip that is additive manufactured one for exact use can take out the total mistakes presented from simple intuition taking and the ensuing thermoset work process $[1,8]$. Notwithstanding more prominent exactness, direct printing produces different advantages, for example, more limited supply chains, fundamentally more limited lead times, and lower costs. It is likewise a more economical measure that produces fundamentally minute waste than additive and thermosettable measures $[1,10]$.

Three-Dimensional manufacturing could be likewise utilized for 3D manufacturing of plain Orthodontic detachable clips by a solitary preparing step utilizing one or a mix of 3D-printing measures [7]. Regardless of the current enhancements and expanded utilization of various sustainable manufacturing advancements, be witnessed by something a restricted count of distributions can be brought into play depicting the immediate additive manufactured endodontic detachable plain clips, or examination with respect to reasonable materials for such printing.

Hypothetically, different 3D printing cycles might be utilized for direct printed clear aligners, for example, combined fiber creation (FFF), particular laser sintering (SLS) or dissolving (SLM), stereolithography (SLA), multi-stream photocured polymer measure, HP MultiJet Fusion innovation or nonstop fluid interface creation innovation [6]. In any case, due to explicit qualities of 
Orthodontic plain aligners and explicit necessities on aligner element charecteristics and execution, threedimensional printing by photograph alkalization from plain gum appears like the most suitable choice.

While there are various enrolled licenses portraying this chance, there are a couple of as of late distributed articles $[5,8,10]$ that have showed up with the main organizations zeroing in on straightforwardly printed aligners and materials for 3D printing. An examination led by Jindal et al. [8] announced an effectively threedimensional manufactured $0.749-\mathrm{mm}$ wide plain detachable clip utilizing endodontic LT $\AA$ (Long Term) plain gum (Form Labs, Somerville, MA, USA) and thought about its traditional and mathematical properties to a routinely fabricated thermosettable dental plain detachable clips assemble of elastic transparent foils. The creators recommended that additive manufactured resin based detachable clips are highly reasonable for customer use as they are mathematically exact; moreover, they can oppose additional greatest burden with a low dislodging, furthermore, can twist flexibly with reversable actions for lower removals. Jindal et al. moreover revealed that LT clear tar has been discovered to be similar with Duran and Durasoft (Scheu-Dental $\mathrm{GmbH}$, Iserlohn, Germany) thermosettable materials under the traditional stress of non-direct push powers comparable to people nibble cycles $[1,11]$; it is implies that sustainable manufacturing has adequate continuous solidarity to oppose outer stacking across a diminishing in medical exhibition.

Nonetheless, Dental LT tar is an affirmed Class IIa biomedical elements with large protection from fracture and it is absolute for gnathological supports, endodontic retainers, and another inflexible exact manufactured endodontic apparatuses as practical one. Its use for additive manufactured detachable clips has not revealed previously, and all creators overlooking the primary limit of the others examinations, or rather the absence of medical information to assess the presentation of Dental LT sap and its opposition during its utilization in the oral pit of customers $[1,12]$.

Progressively, various primer investigations in regard to additive manufactured detachable clips have been done in the regular stages. As recently referenced concerning thermosettable detachable clips, the Wideness of the clip impacts the size of power it conveys to produce ideal teeth development and execution. Sustainable manufacturing addresses an unpredictable technique in dealing the wideness of endodontic clips and in this way the applied power. Edelman et al. revealed an increment of generally wideness than the comparing configuration record, particularly with LT clear pitches [1,13]. The outcomes acquired in the investigation explained that the additive manufacturing work process didn't decisively understand the planned wideness with plain material, and it might gives harmful impacts of medical usage of clips.

\section{Biomedical materials for orthodontics}

There are numerous reasonable saps utilized for sustainable manufacturing of apparatuses in endodontistry, however no clear tar is focused on additive manufactured detachable clips. In mid-2018 (EnvisionTEC Inc., Dearborn, MI, USA) reported the business arrival of E-Ortholign, an imaginative element for the immediate additive manufactured detachable clips [1]. Notwithstanding, this unmistakable tar is expressed as a microporous, compactly steady, adaptable, and solid material for the immediate additive manufacturing of what is referred to in endodontics as a "first aligner" - a follower that is utilized after an endodontist eliminates a customer's sections will gives grip to teeth, set up initially the care is finished with a progression of expectedly made thermoformable aligners $[1,14]$. These days, there is no promoted and endorsed photopolymerizable sap appropriate for the immediate printing of tooth plain clips yet an expanding interest in the field of endodontics is occurring, particularly for the improvement of ensured biocompatible saps [15]. Notwithstanding the absence of existing biocompatible gums for three-dimensional direct plain aligners, some test preliminaries have been completed on intentional patients with altered tars and post-printing conventions, be that as it may, clinical results should be affirmed preceding logical distributing (Figure 3) [1].

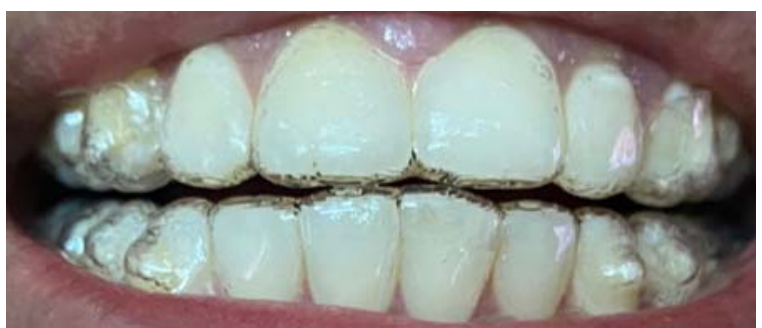

Figure 3. Final step of an endodontic treatment with additive manufactured plain aligners (experimental trial on a voluntary patient).

Moreover, as far as anyone is concerned, while there are a few CAD programming bundles for treatment arranging and arrangement of Three-dimensional designs for aligner forming, just Maestro 3D (Ortho Studio v.5.2, AGE Solutions S.r.1., Pontedera, Italy) has an extra module that can likewise plan aligners and fare them in an arrangement helpful for plain additive manufacturing. Much appreciated to post-trade CAD programming, it is additionally conceivable to completely tweak the wideness of additive manufactured detachable plain clips to apply particular and powers (Figure 4) [1]. 


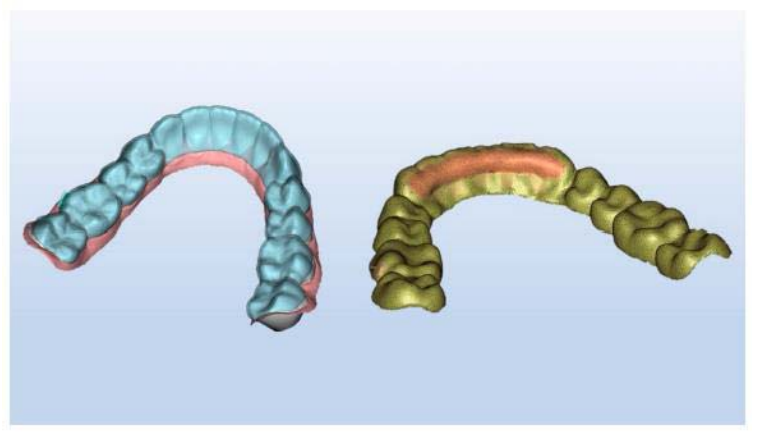

Figure 4. Customization of design and thickness of detachable aligners.

Equitably, the immediate 3D printing of aligners offers a few benefits over regular manufacture, simply using similar computerized includes at present accessible for development of chomp supports:

Borders are carefully planned and indistinguishably imitated for every arrangement of plain removals.

Aligner rims are soft and needn't bother with managing or cleaning.

Undermines don't exist since they are carefully characterized.

Plain removals are manufactured with deeper exactness as aligners are no blunders presented. Throughout the time of printing of a three-dimensional trim model and thermal phase of creation.

Deeper accuracy prompts best fixing and higher viability.

Intra-plain aligners compactness is adaptable, and it may lessen the requirement for connections, which for the most part bring down the straightforwardness of clear aligners.

Given these qualities, when a specially appointed sap is free on the lookout, straightforwardly 3Dprinted aligners should be tried clinically to show their biomechanical viability. Various plans ought to be presumably utilized the assembling of detachable clips contrasted with thermosettable ones because of the chance of making adaptable wideness and connections; traditional properties of adjustable clips in regard to the materials, thickness, and helper components can likewise be examined by invariable demonstrating and limited component investigation $[1,16]$.

\section{Conclusions}

Through Sustainable manufacturing 3D Printed components is utilized in various Domains of endodontist. Three-dimensional Manufacturing innovation is a reasonable strategy for creation of plain aligners and gives a few benefits than the regular thermoformable measure. While the present status of distributed writing about straightforwardly 3D Manufactured plain aligners displays that such an interaction is conceivable, no affirmed element advertised for this reason exists, and programming custom fitted to this point must be created. Hence, no medical investigations of detachable plain clips could be found. In view of these areas, further in glass and in viable considers are expected to check the trended advances and elements. Uncommon consideration should get hold of conveying the cytostatic of sustainable pitches and clinical exhibitions of sustainable manufactured detachable clips should determine in contrast the acquired information and these thermoformable clips. Dentistry has certainly gone in another added substance time.

\section{References}

1. Gianluca M. Tartaglia, Andrea Mapelli, Cinzia Maspero, Tommaso Santaniello, Marco Serafin, Marco Farronato, Alberto Caprioglio. Direct $3 D$ Printing of Clear Orthodontic Aligners: Current State and Future Possibilities, Materials, (2021).

2. Zinelis, S.; Eliades, T.; Eliades, G.; Makou, M.; Silikas, N. Comparative assessment of the roughness, hardness, and wear resistance of aesthetic bracket materials. Dent. Mater., 21, 890-894, (2005).

3. Dobrin, R.J.; Kamel, I.L.; Musich, D.R. Loaddeformation characteristics of polycarbonate orthodontic brackets. Am. J. Orthod, 67, 24-33, (1975).

4. Kaur, S.; Singh, R.; Soni, S.; Garg, V.; Kaur, M. Esthetic orthodontic appliances-A review. Ann. Geriatr. Educ. Med. Sci., 5,11-14, (2008).

5. Leonardi, R. Cone-beam computed tomography and three-dimensional orthodontics. Where we are and future perspectives. J Orthod., 46, 4548, (2019).

6. Shivapuja, P.; Shah, D.; Shah, N.; Shah, S. Direct 3D-Printed Orthodontic Aligners with Torque, Rotation, and Full Control Anchors. U.S. Patent No. 10,179,035, (2019).

7. Prasad, S.; Kader, N.A.; Sujath, G.; Raj, T. $3 D$ printing in dentistry. J. 3D Print. Med., 2, 8991, (2018).

8. Nguyen, T.; Jackson, T. $3 D$ technologies for precision in orthodontics. Semin. Orthod., 24, 386-392, (2018).

9. Jindal, P.; Juneja, M.; Siena, F.L.; Bajaj, D.; Breedon, P. Mechanical and geometric properties of thermoformed and $3 D$ printed clear dental aligners. Am. J. Orthod. Dentofacial. Orthop., 156, 694- 701, (2019).

10. Peeters, B.; Kiratli, N.; Semeijn, J. A barrier analysis for distributed recycling of $3 D$ printing waste: Taking the maker movement perspective. J. Clean. Prod., 241, 118313, (2019).

11. Mohnish Kumar, S. Cytotoxicity of $3 D$ Printed Materials: An In Vitro Study, Sri Ramakrishna Dental College and Hospital: Coimbatore, India, (2019).

12. Jindal, P.; Worcester, F.; Siena, F.L.; Forbes, C.; Juneja, M.; Breedon, P. Mechanical behaviour of $3 D$ printed vs thermoformed clear 
dental aligner materials under non-linear compressive loading using FEM. J. Mech. Behav. Biomed. Mater., 112, 104045, (2020).

13. Maspero, C.; Tartaglia, G.M. $3 D$ Printing of Clear Orthodontic Aligners: Where We Are and Where We Are Going. Materials, 13, 5204, (2020).

14. Edelmann, A.; English, J.D.; Chen, S.J.; Kasper, F.K. Analysis of the thickness of 3dimensional-printed orthodontic aligners. Am. J. Orthod. Dentofac. Orthop., 158, e91-e98, (2020).

15. EnvisionTEC. Available online: https://envisiontec.com/orthodontic-materialslaunched-at-lmt-lab-day-chicago (accessed on 11 November 2019).

16. Nakano, H.; Kato, R.; Kakami, C.; Okamoto, H.; Mamada, K.; Maki, K. Development of Biocompatible Resins for 3D Printing of Direct Aligners. JPST, 32, 209-216, (2019). 